\title{
CRITICALITY DEPENDENCE ON DATA AND PARAMETERS FOR A PROBLEM IN COMBUSTION THEORY, WITH TEMPERATURE-DEPENDENT CONDUCTIVITY
}

\author{
K. K. TAM ${ }^{1}$
}

(Received 22 July 1986; revised 29 September 1988)

\begin{abstract}
A problem in combustion theory with tenperature-dependent conductivity is considered. It is shown that information regarding criticality dependence on data and parameters can be obtained from a transformed equation in which the conductivity is constant, while the nonlinear source term is modified. Some previous work can then be used in such a study.
\end{abstract}

\section{Introduction}

A central problem in combustion theory is the initial and boundary value problem (IBVP) consisting of the nonlinear parabolic equation

$$
\partial \theta / \partial t=\nabla^{2} \theta+\delta f(\theta), \quad \mathbf{x} \in D, t>0,
$$

where $f(\theta)=\exp (\alpha \theta /(\alpha+\theta))$, and the conditions

$$
\theta(\mathbf{x}, 0)=h(\mathbf{x}) \geq 0, \quad \mathbf{x} \in D,
$$

and

$$
\theta(\mathbf{x}, t)=0, \quad \mathbf{x} \text { on } \partial D, t>0 .
$$

Here, $\theta$ is the temperature, $\mathbf{x}$ and $t$ are the nondimensional spatial and time variables, $\delta$ is a parameter incorporating the chemical properties of the combustible material, the external temperature, as well as its geometrical dimensions, and $\alpha$ is a parameter related to the activation energy of the material occupying the region $D$. Typically, $\alpha$ is considerably greater than unity.

\footnotetext{
${ }^{1}$ Department of Mathematics and Statistics, McGill University, Montreal, Canada H3A 2K6

(C) Copyright Australian Mathematical Society 1989, Serial-fee code 0334-2700/89
} 
A number of investigators have studied this problem. The derivation of (1.1) and reference to the early work can be found in [1]. Recently, Tam [4] used a two-step linearisation procedure to study the criticality dependence of the solution and data and parameters. In (1.1), the coefficient of thermal diffusion has been taken as constant. If this coefficient is in fact dependent on the temperature, then the relevant equation to be studied is

$$
\partial \theta / \partial t=\nabla \cdot(k(\theta) \nabla \theta)+\delta f(\theta),
$$

where $k(\theta)$ has the property $k(0)=1, k^{\prime}(\theta)>0$. Typical examples are $k(\theta)=1+b \theta$, and $k(\theta)=\exp (b \theta)$, where $b$ is a positive parameter. In this note, we examine the influence of such a temperature-dependent diffusion coefficient on the parameter and data dependence of the solution. Indeed, the influence of $k(\theta)$ on the critical value of $\delta$, based on the steady state equation, has been carried out by Lacey and Wake [2], and Zaturska and Banks [5], among others. Our objective is to show that the desired information can be obtained from the study of an equation similar to (1.1), in which the nonlinear coefficient $k(\theta)$ is transferred from the diffusive term to the source term. Then the procedure developed in [4] can be effectively used. This is done in Section 2, under certain restrictions on $h(\mathbf{x})$. In Section 3, we compute the critical value of $\delta$ for the sphere, for $k(\theta)=e^{b \theta}$ for different values of $b$, and obtain the threshold value of $b$ above which criticality is lost.

\section{The main result}

We first introduce the transformation

$$
u=\int_{0}^{\theta} k(s) d s
$$

Equation (1.4) then becomes

$$
\partial u / \partial t=K(u)\left\{\nabla^{2} u+\delta F(u)\right\},
$$

where we have written $k(\theta(u)) \equiv K(u)$ and $f(\theta(u)) \equiv F(u)$. We observe that $K(u)>0, K^{\prime}(u)>0$, and $F(u)$ has the same feature as $f(\theta)$. In terms of $u$, the initial and boundary conditions are

$$
u(\mathbf{x}, 0)=H(\mathbf{x})=\int_{0}^{h(\mathbf{x})} k(s) d s
$$

and $u=0, \mathrm{x}$ on $\partial D$. 
LEMMA A. Let $u(\mathbf{x}, t)$ be the solution of the IBVP

$$
\partial u / \partial t=K(u)\left\{\nabla^{2} u+\delta F(u)\right\}
$$

$u(\mathbf{x}, 0)=H(\mathbf{x}), u=0$ on $\partial D$ where $H(\mathbf{x})$ is such that $\nabla^{2} H+\delta F(H)>0$, then $\partial u / \partial t \geq 0$ for $0<t<\infty$.

Proof. It is clear that $(\partial u / \partial t)(x, 0)>0$. We wish to show that $\partial u / \partial t$ does not become negative. Let $t=t_{1}$ be the first instant when $\partial u / \partial t=0$ at some point $\mathbf{x}$. (The situation $(\partial u / \partial t)\left(\mathbf{x}, t_{1}\right)=0$ for all $\mathbf{x}$ in $D$ need not be considered, for then an equilibrium value of $u$ will have been reached.) In a neighbourhood of $\mathbf{x}_{1}$, we have $\partial u / \partial t>0$, and hence

$$
\begin{gathered}
u\left(\mathbf{x}_{1}, t_{1}+\varepsilon\right)=u\left(\mathbf{x}_{1}, t_{1}\right)+0\left(\varepsilon^{2}\right), \\
u\left(\mathbf{x}, t_{1}+\varepsilon\right)=u\left(\mathbf{x}, t_{1}\right)+0(\varepsilon), \quad \mathbf{x} \neq \mathbf{x}_{1} .
\end{gathered}
$$

Recalling that the limiting value of the difference between $u$ at a point $\mathbf{x}$ and the average value of $u$ in a neighbourhood of $\mathbf{x}$ is proportional to $-\nabla^{2} u$ (see [3] p. 7), we see that $\nabla^{2} u\left(\mathbf{x}_{1}, t_{1}+\varepsilon\right)>\nabla^{2} u\left(\mathbf{x}_{1}, t_{1}\right)$. Thus, we have

$$
\frac{\partial^{2} u\left(\mathbf{x}_{1}, t_{1}\right)}{\partial t^{2}}=K\left(u\left(\mathbf{x}_{1}, t_{1}\right)\right)\left\{\frac{\partial}{\partial t} \nabla^{2} u\left(\mathbf{x}_{1}, t_{1}\right)\right\}>0,
$$

implying $\partial u / \partial t$ does not become negative. The same conclusion holds if $\partial u / \partial t$ vanishes at a number of points instead of at a single point. (The author is thankful to a referee who pointed out that the above result follows by applying the maximum principle to the time derivative of the equation for $u$.)

LEMMA B. Let $u, V$ and $v$ respectively be the solution of the IBVP

$$
\begin{aligned}
& P u=\frac{\partial u}{\partial t}-K(u)\left\{\nabla^{2} u+\delta F(u)\right\}=0, \\
& \frac{\partial V}{\partial t}=\left\{\max _{\mathbf{x} \in D} K(V)\right\}\left\{\nabla^{2} V+\delta F(V)\right\}, \\
& \frac{\partial v}{\partial t}=\left\{\max _{\mathbf{x} \in D} K(v)\right\}\left\{\nabla^{2} v+\delta F(v)\right\},
\end{aligned}
$$

subject to the same initial condition $u(\mathbf{x}, 0)=V(\mathbf{x}, 0)=v(\mathbf{x}, 0)=H(\xi)$, and boundary condition $u=V=v=0$ on $\partial D$, where $H(\mathbf{x})$ is such that $\nabla^{2} H+\delta F(H)>0$. Then we have $v \leq u \leq V$.

Proof. The same argument in the proof of Lemma A shows that $\partial V / \partial t=$ $\max _{\mathbf{x} \in D} K(V)\left\{\nabla^{2} V+\delta F(V)\right\} \geq 0$ for $0<t<\infty$. Then, since $P V=\partial V / \partial t-$ $K(V)\left\{\nabla^{2} V+\delta F(V)\right\}=\left\{\max _{\mathbf{x} \in D} K(V)-K(V)\right\}\left\{\nabla^{2} V+\delta F(V)\right\}$ we have $P V \geq 0$, implying $u \leq V$. In the same way, we have $P v \leq 0$, implying $v \leq u$. 
COROLlary. $V$ and $v$, and hence $u$, tend to the same asymptotic state as $t \rightarrow \infty$.

Proof. Let $\bar{D}(t ; V)=\max _{\mathbf{x} \in D} K(V)$. If we make the transformation $\tau(t)=$ $\int_{0}^{t} \bar{D}(s ; V) d s$, equation (2.4) is transformed into $\partial V / \partial \tau=\nabla^{2} V+\delta F(V)$. Similarly, if we denote $\tilde{D}(t ; v)=\min _{\mathrm{x} \in D} K(v)$, and make the transformation $\eta(t)=\int_{0}^{t} \tilde{D}(s ; v) d s$, equation $(2.5)$ is transformed into $\partial v / \partial \eta=\nabla^{2} v+$ $\delta F(v)$. Thus, $V$ and $v$ tend to the same asymptotic state. Since $v \leq u \leq V$, so does $u$.

REMARK. It is clear that the steady states of (2.3), (2.4) and (2.5) are all given by the solution of $\nabla^{2} u+\delta F(u)=0, u=0$ on $\partial D$. If there is a unique steady state, then the Corollary is obvious. However, if there are more than one steady states, then Lemma B and its Corollary assert that the same initial condition leads to the same steady state.

Thus, to study the influence of $\delta$ and $h(\mathbf{x})$ on the asymptotic solution of (1.4), it suffices to study the influence of $\delta$ and $H(\mathbf{x})$ on the asymptotic solution of

$$
\partial u / \partial t=\nabla^{2} u+\delta F(u)
$$

\section{An example}

Since the criticality dependence on initial data and parameter can be studied through (2.6), the procedure developed in [4] can be used. In this note, we shall not carry out extensive computation for various domains, of Class $\mathrm{A}$ or otherwise. Rather, we shall do one example as an illustration. Specifically, we consider the sphere with $k(\theta)=e^{b \theta}, \alpha=100, h(x)=0$, and compute the critical values of $\delta$ for various values of $b$. To proceed as in [4], we consider the transcendental equation

$$
\lambda_{1}^{2} T / \delta=\int_{D} \phi_{1}(\mathbf{x}) F\left(T \phi_{1}(\mathbf{x})\right) d v
$$

where $\lambda_{1}^{2}$ and $\phi_{1}(x)$ are respectively the first eigenvalue and normalised eigenfunction of the equation $\nabla^{2} \phi_{1}=-\lambda_{1}^{2} \phi_{1}, \phi_{1}=0$ on $\partial D$. Plotted against $T$, the term on the right side of (3.1) is an $s$-shaped curve. The critical value of $\delta$ is that particular value when the straight line $\lambda_{1}^{2} T / \delta$ is tangent to the $s$-shaped curve where its curvature is positive. For the sphere, we have $\lambda_{1}^{2}=\pi^{2}, \phi_{1}=\sin \pi r / \sqrt{2 \pi} r$. With $k(\theta)=e^{b \theta}$, we have

$$
\begin{gathered}
b \theta=\ln (1+b u), \\
F(u)=\exp \{\alpha \ln (1+b u) /(b \alpha+\ln (1+b u))\} .
\end{gathered}
$$


The results are tabulated in Table I. In [2] Lacey and Wake considered the simpler equation $\nabla \cdot\left(e^{b \theta} \nabla \theta\right)+\delta e^{\theta}=0$ and showed that for $b \geq 1$, the solution exhibits no criticality phenomemon. Further, $\delta_{\text {cr }}$ tends to the first eigenvalue as $b$ tends to unity from below. In our case, this loss of criticality occurs at $b=0.9$ and $\delta_{\text {cr }}$ tends to $0.846 \lambda_{1}^{2}$.

TABLE I. $\delta_{\mathrm{cr}}$ as a function of $b$ for $k(\theta=\exp (b \theta), \alpha=100$.

\begin{tabular}{|c|c|c|c|c|c|}
\hline$b$ & 0.1 & 0.2 & 0.4 & 0.7 & 0.8 \\
\hline$\delta_{\mathrm{cr}}$ & 3.564 & 3.902 & 4.349 & 5.922 & 6.779 \\
\hline
\end{tabular}

\section{Concluding remarks}

We have cast equation (1.4), which has temperature dependent conductivity, into an equation amenable to treatment by a method developed earlier. Questions such as dependence on parameters, initial condition, and various boundary conditions can be addressed in the same unified manner as for an equation with constant conductivity. Thus we have extended the scope of the previous results.

\section{Acknowledgement}

This research was supported by the Natural Science and Engineering Research Council of Canada.

\section{References}

[1] D. A. Frank-Kamenetskii, Diffusion and heat transfer in chemical kinetics (Translat. editor J. P. Appleton), (Plenum Press, New York, 1959).

[2] A. A. Lacey and G. C. Wake, "Thermal ignition with variable thermal conductivity", IMA J. Appl. Math. 28 (1982) 23-39.

[3] P. M. Morse and H. Feshbach, Methods of Theoretical Physics (McGraw-Hill, New York, 1953).

[4] K. K. Tam. "Criticality dependence on data and parameters for a problem in combustion theory", J. Austral. Math. Soc. Ser. B 27 (1986) 426-441.

[5] M. B. Zaturska and W. H. H. Banks, "Thermal explosion with variable thermal conductivity: criticality and its disappearance", IMA J. Appl. Math. 34 (1985) 73-82. 